\title{
Riesgo de muerte por suicidio en población Colombiana 2000-2013
}

\author{
The risk of death by suicide in the Colombian population \\ 2000-2013
}

Jorge Martín Rodríguez-Hernández ${ }^{1}$

Anderson Rocha-Buelvas ${ }^{2}$

Giovane Mendieta-Izquierdo ${ }^{3}$

Arsenio Hidalgo-Troya ${ }^{4}$

${ }^{1}$ Instituto de Salud Pública, Pontificia Universidad Javeriana. Carrera 7 No. 40. Bogotá Colômbia. jrodriguez.h@ javeriana.edu.co

${ }^{2}$ Facultad de Ciencias de la Salud, Universidad de

Nariño. Pasto Colômbia.

${ }^{3}$ Facultad de Educación y

Humanidades, Universidad

Militar Nueva Granada.

Bogotá Colômbia.

${ }^{4}$ Investigador del Centro

de Estudios en Salud,

Universidad de Nariño.

Pasto Colômbia.
Abstract The scope of this article is to analyze the risk of death by suicide in Colombia based on the covariables of sex, age group, year, region and/ or department from 2000 to 2013. The databases of deaths by suicide and population projections of DANE were used. A longitudinal and analytical study was conducted. From 2000 to 2013, annual trends of deaths by suicide by means of modeling of covariables were established and risk estimates were collected. The Poisson regression model (PRM) was used. IRR was used according to the $M R P$ with a level of significance of $(P<0.05)$. Colombians between the years 2000-2013, male, 15 to 34 years old, from the Central and Eastern regions and from the Departments of Vaupés, Huila and Quindio were those with the highest risk of death by suicide. The suicide rate decreased slightly as the years went by. The highest risk of suicide occurred among men of productive age, older adults, and in underdeveloped regions. Colombia requires a mental health policy that implements strategies for primary care, health promotion and disease prevention, aimed at promoting the quality of life, detection of suicidal ideation, treatment and rehabilitation of these people from a comprehensive rights and care approach.

Key words Suicide, Mortality, Relative risk, Colombia
Resumen Analizar el riesgo de muerte por suicidio en Colombia, a partir de covariables sexo, grupo etario, año, región y/o departamento de 2000 a 2013. Se utilizaron las bases de datos de muertes por suicidio y proyecciones poblacionales del DANE. Se realizó un estudio longitudinal y analitico. De 2000 a 2013, se establecieron las tendencias anuales de muertes por suicidio por covariables mediante modelación y se recogieron estimaciones de riegos Se utilizó el modelo de regresión de Poisson (MRP). Se emplearon IRR según el MRP con nivel de significancia $(P<0,05)$. Los colombianos entre los años 2000-2013, de sexo masculino, de 15 a 34 años, de las regiones Central y Oriental y de los Departamentos de Vaupés, Huila y Quindío fueron los que mayor riesgo tuvieron de morir por suicidio. La tasa de suicidios fue levemente decreciente conforme pasaron los años. El mayor riesgo de suicidio ocurrió en hombres en edad productiva, adultos mayores $y$ en regiones poco desarrolladas. Colombia requiere una política de salud mental que implemente estrategias de atención primaria, promoción de la salud y prevención de la enfermedad, orientadas a fomentar la calidad de vida, detección de ideación suicida, tratamiento y rehabilitación a estas personas desde el enfoque de derechos y atención integral.

Palabras claves Suicidio, Mortalidad, Riesgo relativo, Colombia 


\section{Introducción}

La violencia es una situación de salud pública que ha afectado a América Latina (AL), la magnitud del problema es mayor que en Europa, Estados Unidos y Canadá; según diversas fuentes, la violencia en 2002 para AL estuvo entre 26-30/100.000 habitantes, mientras que en diferentes partes de Europa la tasa de homicidios osciló entre 2-3/100.000 habitantes ${ }^{1}$. En AL, la violencia ha afectado principalmente a los hombres, con una razón $7 / 1$, respecto a las mujeres. Se ha distribuido de forma variable, con Colombia, como el país que encabezó por varios años esta problemática (cerca de 104 homicidios por 100.000 hombres); también países como Salvador (129/100.000 hombres), Guatemala (70,3/100.000 hombres), Venezuela (64/100.000 hombres), Brasil (54/100.00 habitantes) y últimamente México (23/100.000 hombres) son los países que han encabezado las más altas cifras de homicidio en la región ${ }^{2}$.

Dentro de las lesiones y muertes, también se encuentran aquellas de naturaleza autoinflingida, las cuales incluyen desde la ideación suicida hasta el acto consumado que genera la muerte. Según el informe mundial de Salud y Violencia de 2003, para el año 2000, cerca de 815.000 personas murieron por esta causa a nivel mundial; lo anterior comprendía una tasa cercana a 14,5/100.000 habitantes, es decir una muerte por esta causa menos de cada minuto; las tasas han sido variables, donde países Europeos (Austria: 20.9; Bélgica: 24; Croacia: 24.8; Estonia: 37.9; Rusia: 43,6, entre otros) y Asiáticos (Kazajstán: 37,4; China: 18.3), tienen tasas de mortalidad superior a 18/100.0001.

Los comportamientos auto lesivos con incremento de la probabilidad de muerte (intentos de suicidios y suicidio consumado), se han intentado explicar desde la neuropsicología, a partir de una perspectiva conductual y su vínculo con la toma de decisiones y estímulos negativos ${ }^{3}$, la falta de resolución de conflictos, disfunción familiar y consumo de alcohol ${ }^{4}$. Las acciones de autolesión con mayor frecuencia se presentan por consumo de fármacos medicados, es por esto que se sugiere tener especial cuidado con el uso en pacientes en riesgo de autolesión ${ }^{5}$. Desde una perspectiva psicológica se ha estudiado la ideación y el suicidio desde autopsias psicológicas para comprender el contexto sociocultural ${ }^{6}$. El potencial de suicidio puede relacionarse con factores de riesgo para la ansiedad cognitiva ${ }^{7}$. La enfermedad psiquiátrica, es el primero de todos los trastornos afectivos, en especial en el caso de un intento previo de suici- dio, este es el más potente predictor de suicidio. El reconocimiento temprano y tratamiento adecuado de los trastornos psiquiátricos, así como la atención posterior de las personas con un alto riesgo de suicidio son, en consecuencia, los métodos más exitosos de la prevención de este evento ${ }^{8}$.

En 2000 el suicidio se encontraba entre las primeras quince causas de muerte a nivel mundial'; no obstante, está dentro de las primeras diez causas de años ajustados por discapacidad (AVISA) en personas en edades comprendidas entre los 15 a los 44 años. La razón de mortalidad por suicidio ha sido variable, dependiendo del país o región, en algunos países de Europa ha alcanzado razones por sexo de 6 a 6.5: 1 (Eslovaquia, Rusia), mientras que en países Asiáticos, la razón ha sido de 1.5:1. En las Américas, ha sido preocupante la situación en países como Cuba (23/100.000 habitantes), Canadá (15/100.000 habitantes) y USA (13.9/100.000) los cuales tienen valores cercanos o arriba del promedio Mundial ${ }^{1}$.

En Colombia el intento de suicidio tiene una incidencia menor a la de otros países, con tasas de mortalidad de 4.1/100.000 habitantes ${ }^{1}$. La población con mayor riesgo está entre 16 y 21 años, se destaca la depresión o ansiedad, consulta al médico por 'nervios', abuso verbal, diferentes valores morales a los de su familia ${ }^{10}$. El último informe de carga de enfermedad reporta que las primeras veinte causas de años potencialmente perdidos AVISAS se encuentran eventos como trastorno depresivo mayor (segunda causa), trastornos bipolares (séptima causa), esquizofrenia (décima causa), trastornos mentales por uso de drogas (causa veinte) $)^{11}$, los cuales se han identificado como factores de riesgo potencialmente asociados a conductas, comportamientos y acciones suicidas. Analizar el riesgo de muerte por suicidio en Colombia, a partir de covariables sexo, grupo etario, sexo, año, región y/o departamento de 2000 a 2013.

\section{Metodología}

Se realizó un estudio longitudinal y analítico ${ }^{12}$ que estimó el riesgo de muertes por suicidios en el período comprendido entre los años 2000 a 2013 en Colombia. Se emplearon registros de información secundaria; para el presente estudio se usó la base de datos de los registros de defunciones del Departamento Nacional de Estadística -DANE-, del cual se extrajeron las causas de defunción individuales de los eventos clasificados como muertes violentas con los códigos de 
la Clasificación Internacional de EnfermedadesCIE- $10^{13}$, agrupados de la siguiente forma: las variables analizadas fueron: año de ocurrencia de la muerte (2000 al 2013), edad (0 a 99 años), sexo (Masculino o Femenino), grupo de causas del CIE 10 y entidad territorial de ocurrencia de la muerte (departamentos de Colombia). La edad se agrupó generando grupos etarios ( 0 a 14 años o menores de 15 años, 15 a 34 años, 35 a 49 años, 50 a 69 años y 70 y más años), mientras los departamentos se agruparon por regiones geográficas (Caribe e insular, Central, Bogotá-Cundinamarca, Oriental, Pacífica, Amazonia-Orinoquia) ${ }^{14}$.

Se calcularon tasas específicas con las proyecciones de población reportadas por el Departamento Administrativo Nacional de Estadística de Colombia ${ }^{15}$ para cada uno de los años. Todas las tasas se reportan por 100.000 habitantes. Para calcular las tasas se utilizaron las poblaciones estimadas por el DANE en el periodo 2000-2013 ${ }^{15}$ distribuidas por sexo, grupos de edad y región. Se calcularon -TAE- tasas estandarizadas de mortalidad por región y departamento a través del método directo utilizando la población estándar mundial de Segi ${ }^{16}$. La Razón Estandarizada de Mortalidad -REM-, se estimó a partir de las tasas estandarizados de cada departamento entre el promedio nacional ${ }^{17,18}$.

La tendencia de las tasas anuales de suicidio en Colombia, durante el período 2000-2013 se realizaron ajustando un modelo de regresión de Poisson -MRP- ${ }^{19}$, por consiguiente, el número de muertes por suicidio fue modelado como una variable aleatoria de Poisson con la media ( ), tomando como variable dependiente $\mathrm{Y}=\mathrm{Ln}(\mathrm{R})$, donde $\mathrm{R}$ representa las tasas de mortalidad por suicidio y como variables explicativas X1 edad, $\mathrm{X} 2$ sexo, X3 periodo de tiempo y X4 región. Se estimaron los riegos relativos junto con intervalos de confianza al 95\%, para cada una de las categorías de las variables del estudio, tomando como referencia para edad a los menores de 15 años, para sexo a las mujeres, para región las REM Amazonia-Orinoquia y para año cada uno de los años.

El MRP se determinó utilizando el paquete estadístico IBM SPSS versión 22 por medio de la función Modelo Lineal Generalizado -MLG- ${ }^{19}$. Los supuestos más importantes del MRP fueron la equidispersión y la verificación de esta propiedad mediante los estadísticos de desvianza y chi cuadrado de Pearson. Los estadísticos de bondad de ajuste demostraron que los valores escala estimados no indicaron sobredispersión ni violación a esta propiedad ${ }^{20}$, El MRP se definió como: LnR
$=-12,70-0,46 \mathrm{R} \_1+0,30 \mathrm{R} \_2-0,16 \mathrm{R} \_3-+0,23 \mathrm{R} \_4$ $+0,19 \mathrm{R} \_5+1,34 \mathrm{~S} \_1+2,38 \mathrm{E} \_1+2,16 \mathrm{E} \_2+2,16 \mathrm{E}$ _3+2,59E_4-0,03A. La utilización de la prueba Omnibus evidenció que el MRP se ajustó a los datos ( $p$ valor $\leq 0.05$ ), con un valor de medida de ajuste Pseudo $\mathrm{R}^{2}=0,82^{21}$. La población a riesgo para este análisis fue la estimada por DANE en 2006 (mitad de periodo).

\section{Resultados}

Entre el periodo 2000-2013 se reportaron en Colombia 31.053 casos, de estos 24.460 ocurrieron en hombres y 6.593 en mujeres. La tasa de muertes por suicidio en Colombia entre 2000-2013 no varío mucho, siendo la más alta, 5.95 por 100.000 habitantes, en 2001, y la más baja de 4.40 en 2013. El promedio anual de la tasa cruda de suicidios en general fue de 5,9, para una razón de 8,11 (hombres) por 2,13 (mujeres) por 100.000 habitantes. Hay que destacar que se encontró que en los hombres la tasa más alta fue de 9.29 en el año 2001 y la más baja fue de 7.17 en el año 2013; mientras en las mujeres la más alta fue de 2.72 en el año 2003 y la más baja fue de 1.64 en el año 2012. De modo que las tasas en hombres son de manera sistemática superiores a las de las mujeres; siendo la tendencia levemente descendente en los dos grupos (Tabla 1).

La tasa de muertes por suicidio más alta en Colombia entre el periodo de 2000-2013 según grupo de edad fue de 8.48 por 100.000 habitantes, específicamente en el grupo de edad de 15 a 34 años. No obstante, la TAE en hombres fue de 8.03 y en mujeres fue de 2.05; donde los hombres mayores de 70 años poseen la tasa más alta de muertes por suicidio con 13.4 por 100.000 habitantes, siguiéndole el grupo etario de 15 a 34 años con una tasa de 12.8 y con una variación mínima con respecto a los otros grupos de edad, excepto con respecto a los menores de 15 años, cuya tasa es entre 10 y 13 veces menor a los otros grupos de edad. En las mujeres la tasa más alta fue entre los 15-34 años, como en ambos sexos, sin embargo, los otros grupos de edad se expresan diferente a los hombres, ya que dicha tasa fue aproximadamente tres veces mayor que la presentada en los grupos de edad entre 35 a 49 años y entre 50 a 69 años y aproximadamente cuatro veces mayor con respecto a la tasa de muertes por suicidio en los menores de 15 años y los mayores de 70 años (Tabla 2).

Las regiones Central y Oriental son las que tienen mayor riesgo de cometer suicidio en el 
período estudiado con un riesgo superior en un $28 \%$ y $22 \%$ respectivamente, con relación a la TAE nacional promedio. Mientras que la región Caribe/Insular es la región de menor riesgo, inferior en un 37\% al TAE nacional promedio del periodo estudiado (Tabla 3). Por departamentos, Vaupés es el que presenta mayor riesgo de muertes por suicidio en el país entre 2000 a 2013, con una REM 2,16 veces mayor que el promedio nacional; le siguen los departamentos de Huila y Quindío con una REM de 1,71. Mientras que San Andrés y Chocó son los de menor riesgo con un

Tabla 1. Tasas de suicidios por género en Colombia. Periodo 2000-2013 (por 100.000 habitantes).

\begin{tabular}{lrrrrrr}
\hline \multirow{2}{*}{ Año } & \multicolumn{2}{c}{ Total } & \multicolumn{2}{c}{ Mujeres } & \multicolumn{2}{c}{ Hombres } \\
& Casos & Tasa & Casos & Tasa & Casos & Tasa \\
\hline 2000 & 2.383 & 5,92 & 548 & 2,69 & 1.835 & 9,23 \\
2001 & 2.429 & 5,95 & 558 & 2,70 & 1.871 & 9,29 \\
2002 & 2.316 & 5,60 & 550 & 2,63 & 1.766 & 8,66 \\
2003 & 2.287 & 5,47 & 577 & 2,72 & 1.710 & 8,28 \\
2004 & 2.264 & 5,34 & 502 & 2,34 & 1.762 & 8,43 \\
2005 & 2.120 & 4,94 & 467 & 2,15 & 1.653 & 7,81 \\
2006 & 2.188 & 5,04 & 461 & 2,10 & 1.727 & 8,06 \\
2007 & 2.209 & 5,03 & 459 & 2,06 & 1.750 & 8,07 \\
2008 & 2.226 & 5,01 & 449 & 1,99 & 1.777 & 8,10 \\
2009 & 2.261 & 5,03 & 448 & 1,97 & 1.813 & 8,17 \\
2010 & 2.118 & 4,65 & 398 & 1,73 & 1.720 & 7,66 \\
2011 & 2.053 & 4,46 & 384 & 1,65 & 1.669 & 7,34 \\
2012 & 2.125 & 4,56 & 387 & 1,64 & 1.738 & 7,56 \\
2013 & 2.074 & 4,40 & 405 & 1,70 & 1.669 & 7,17 \\
Total & 31.053 & 5,08 & 6.593 & 2,13 & 24.460 & 8,11 \\
Periodo & & & & & & \\
\hline
\end{tabular}

Tabla 2. Tasas de suicidios por edad y género en Colombia. Periodo 2000-2013 (por 100.000 habitantes).

\begin{tabular}{lrrrrrrr}
\hline $\begin{array}{l}\text { Grupos de } \\
\text { edad }\end{array}$ & \multicolumn{2}{c}{ Total } & \multicolumn{2}{c}{ Mujeres } & \multicolumn{2}{c}{ Hombres } \\
\cline { 2 - 7 } & Casos & Tasa & Casos & Tasa & Casos & Tasa \\
\hline $\begin{array}{l}\text { Menor de } \\
15 \text { años }\end{array}$ & 1.203 & 0,65 & 583 & 0,64 & 620 & 0,65 \\
$\begin{array}{l}\text { De 15 a 34 } \\
\text { años }\end{array}$ & 17.311 & 8,48 & 4.305 & 4,20 & 13.006 & 12,80 \\
$\begin{array}{l}\text { De 35 a 49 } \\
\text { años }\end{array}$ & 6.262 & 5,37 & 964 & 1,59 & 5.298 & 9,48 \\
$\begin{array}{l}\text { De 50 a 69 } \\
\text { años }\end{array}$ & 4.471 & 5,56 & 593 & 1,41 & 3.878 & 10,10 \\
$\begin{array}{l}\text { De 70 y } \\
\text { más años }\end{array}$ & 1.628 & 6,51 & 129 & 0,93 & 1.499 & 13,40 \\
Total & 31.053 & 5,08 & 6.593 & 2,13 & 24.460 & 8,11 \\
\hline
\end{tabular}

REM equivalentes de 0,36 y 0.35 respectivamente (Figura 1).

En el Tabla 4 se observa la estimación de los coeficientes del MRP, lo cual permitió establecer que las variables sexo, edad, región y año del evento presentaron asociación estadística significativa ( $\mathrm{p}$ valor $\leq 0.05$ ) con las tasas de muerte por suicidio. El riesgo de muerte por suicidio de los hombres colombianos fue 3.9 veces mayor que el de las mujeres colombianas. En cuanto a la edad, se encontró que los colombianos pertenecientes al grupo de edad de 15 a 34 años, presentaron 13.2 veces mayor riesgo de muerte por suicidio que el grupo de menores de 15 años. Mientras que las regiones que presentaron mayor riesgo de suicidio fueron Central y Oriental (superiores en un 34\% y 25\% respectivamente), le siguen Amazonía-Orinoquía y Pacífico con riesgos estadísticamente similares, y Bogotá-Cundinamarca y Caribe-Insular con los riesgos más bajos (inferiores en un 17\% y $37 \%$ respecto al grupo de referencia). Las diferencias en las tasas de muerte por suicidio por año también resultaron significativas, mostrando una tasa anual de cambio decreciente en un promedio de $3 \%$.

\section{Discusión}

Los resultados de este estudio demuestran que el mayor riesgo de muerte por suicidio en Colombia ocurre en las zonas céntricas y oriental del territorio nacional, en hombres, adultos en edad productiva y en adultos mayores. Estos resultados concuerdan con varios estudios de la literatura internacional, donde ser hombre, soltero, vivir en localidades medianas aumenta el riesgo y probabilidad de conductas suicidas ${ }^{22-24}$.

Tabla 3. Tasas de suicidios por región en Colombia. Periodo 2000-2013 (por 100.000 habitantes).

\begin{tabular}{lcc}
\hline \multicolumn{1}{c}{ Región } & TC & TAE \\
\hline Caribe/Insular & 3,02 & 3,11 \\
Central & 6,54 & 6,33 \\
Bogotá-Cundinamarca & 4,12 & 3,91 \\
Oriental & 6,07 & 6,03 \\
Pacífica & 5,76 & 5,68 \\
Amazonía-Orinoquía & 4,76 & 4,69 \\
Total & 5,08 & 4,95 \\
\hline
\end{tabular}




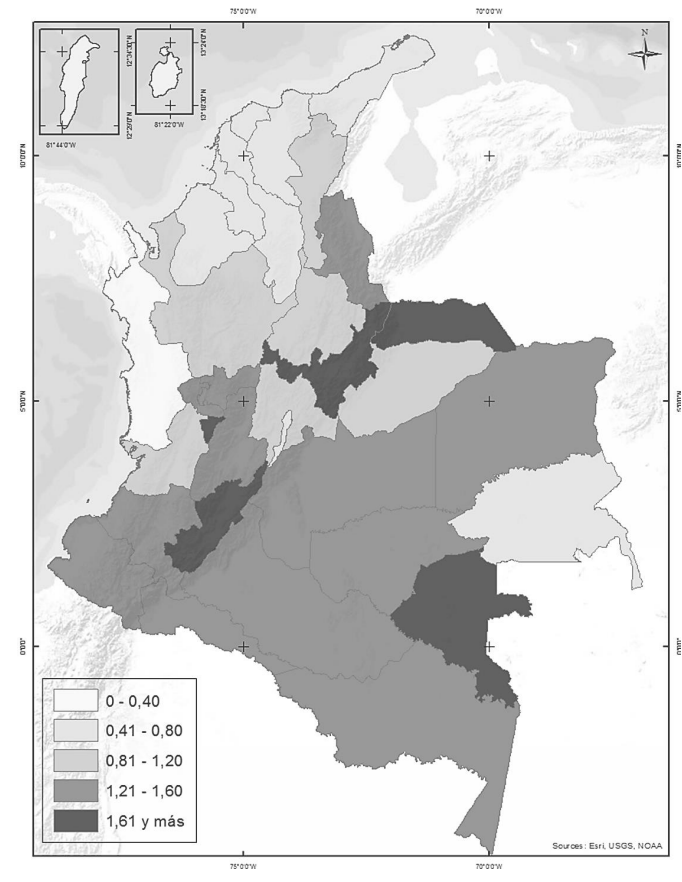

Figura 1. Razones estandarizadas de mortalidad por suicidio por departamento. Colombia 2000 a 2013.
Tabla 4. Estimaciones del riesgo de muertes por suicidio en Colombia 2000-2013 según Modelo de Regresión de Poisson.

\begin{tabular}{|c|c|c|c|c|}
\hline \multirow{2}{*}{ Parámetros } & \multirow{2}{*}{$\begin{array}{c}\mathrm{p} \\
\text { valor }\end{array}$} & \multirow{2}{*}{ RT } & \multicolumn{2}{|c|}{ IC } \\
\hline & & & Inferior & Superior \\
\hline (Interceptación) & 0,001 & 0,000 & $2,514 \mathrm{E}-06$ & $3,823 \mathrm{E}-06$ \\
\hline $\begin{array}{l}\mathrm{R}_{1}=\text { Caribe/ } \\
\text { Insular }\end{array}$ & 0,001 & ,63 &, 53 & ,76 \\
\hline $\mathrm{R}_{2}=$ Central & 0,001 & 1,34 & 1,12 & 1,59 \\
\hline $\begin{array}{l}\mathrm{R}_{3}=\text { Bogotá- } \\
\text { Cundinamarca }\end{array}$ & 0,045 &, 83 & ,70 & 1,00 \\
\hline $\mathrm{R}_{4}=$ Oriental & 0,015 & 1,25 & 1,04 & 1,49 \\
\hline $\mathrm{R}_{5}=$ Pacífica & 0,072 & 1,18 & ,99 & 1,41 \\
\hline $\begin{array}{l}\mathrm{R}_{6}=\text { Amazonía- } \\
\text { Orinoquía }\end{array}$ & & $1,00^{(\mathrm{a})}$ & & \\
\hline$S_{1}=$ Hombres & 0,001 & 3,86 & 3,66 & 4,07 \\
\hline $\mathrm{S}_{2}=$ Mujeres & & $1,00^{(\mathrm{a})}$ & & \\
\hline $\mathrm{E}_{1}=70$ o más & 0,001 & 10,50 & 9,08 & 12,15 \\
\hline $\mathrm{E} 2=50$ a 69 & 0,001 & 8,75 & 7,73 & 9,91 \\
\hline $\mathrm{E}_{3}=35$ a 49 & 0,001 & 8,70 & 7,71 & 9,81 \\
\hline $\mathrm{E}_{4}=15$ a 34 & 0,001 & 13,23 & 11,80 & 14,83 \\
\hline $\mathrm{E}_{5}=$ menor de 15 & & $1,00^{(\mathrm{a})}$ & & \\
\hline $\begin{array}{l}\text { A = Año evento } \\
\text { (Escala) }\end{array}$ & 0,001 & ,97 & ,97 & ,98 \\
\hline
\end{tabular}

Se han generado otras explicaciones para comprender el mayor riesgo de suicidio en personas de mediana edad en regiones con menores zonas urbanas y con menor desarrollo, siendo la más aceptada, la tendencia del suicidio cuando hay desempleo, recesión económica ${ }^{31}$ y consumo de alcohol ${ }^{32}$. En Colombia entre los años 1994 y 2013 se encontró asociación entre desigualdad y suicidio $^{33}$, sin embargo, en una investigación de diseño ecológico, se concluyó que para 2012, la tasa de suicidio fué independiente de personas en situaciones de precariedad y pobreza ${ }^{34}$; no obstante, reconoce que su estudio adolece de los problemas metodológicos de los diseños ecológicos, y en efecto, no tuvieron la posibilidad de identificar y ajustar por otras variables de orden demográfico o social que se hubiera comportado como un factor de confusión entre el indice Gini y las tasas de suicidio ${ }^{35}$.

Con relación a los MRP, existen al menos dos estudios del contexto nacional los cuales realizan estimaciones de riesgo de conductas violentas. El primero utilizó el MRP a partir de covariables similares a las de este estudio, tales como grupo pertenencia a un grupo determinado ${ }^{29,30}$. 
etario y sexo, mostrando que el mayor riesgo de homicidio se encontraba en hombres de edades entre 20 y 30 años $^{36}$. El otro estudio hizo un análisis de suicidio de 1998-2012 sin utilizar MRP; entre los principales hallazgos mostró a partir del Índice de Moran Global, un grado de autocorrelación espacial negativa, es decir hubo municipios con altas tasas de suicidio rodeados por municipios con bajas tasas; también evidenció que el mayor riesgo de suicidio se daba en población joven adulta, masculina y en hombres adultos mayores $^{37}$.

La situación del riesgo de muertes por suicidio en Colombia que afecta a los adultos jóvenes se podría hipotetizar que éstos son más proclives a padecer trastornos de estrés postraumático, padecer de trastornos depresivos, consumo de sustancias psicotrópicas y psicoactivas, y a los antecedentes de intentos de suicidio; todas estas características aumentan el riesgo de muertes auto-inflingidas, teniendo en cuenta que estás circunstancias afectan el funcionamiento familiar, económico y social de los individuos ${ }^{38}$. También se ha descrito la asociación entre discriminación y estigma en sujetos con trastorno mental como factor de riesgo de suicidio ${ }^{39}$.

Otra situación importante alrededor del riesgo de muertes por suicidio, es la que se refiere a la evidencia que las conductas tentativas de suicidio como ideación e intento de suicidio preceden a la consumación. En Colombia 7,6\% de las mujeres idean suicidarse vs 5,5\% de los hombres; $2,7 \%$ de ellas planean suicidarse, mientras que $2,0 \%$ de los hombres lo hacen; así mismo 3,3\% de las mujeres intentan suicidarse vs. $1,9 \%$; de los hombres. Sin embargo son los hombres los que más cometen el suicidio. Por otro lado, varios departamentos con altos riesgos de suicidio, son en los que se observan las mayores conductas de intento de suicidio, por ejemplo los de las regiones Central y Oriental, quienes tienen los mayores intentos de suicidio; de la misma forma, la región Caribe/Insular fue la de más baja proporción de intentos suicidas con menor riesgo de muerte por suicidio ${ }^{38}$.

Las lesiones autoinflingidas son un reto para la salud pública, estas estas son comprendidas como una situación evitable; así como muchos otros problemas de salud que incrementan la carga de enfermedad, los costos de atención directa al sistema de salud e indirectos a la familia, aspecto que se ha estimado anualmente a nivel mundial $^{40}$. El suicidio, como manifestación de violencia auto-inflingida, es un problema de salud, donde los profesionales de atención primaria pueden y deben ayudar a enfrentar la problemáti- ca. Se ha observado que los pacientes con estados depresivos, visitan con frecuencia los servicios de salud, las semanas y meses anteriores al "suicidio exitoso" 41 . Investigaciones han mostrado que existe una mejora sustancial en el manejo de la depresión y el descenso de la probabilidad de suicidio con la participación de la atención prima$\mathrm{ria}^{42}$. Desde la OMS, el suicidio se considera un imperativo global, es así como se han establecido lineamientos y estrategias para la formulación de políticas públicas para su prevención ${ }^{43}$.

El presente estudio destaca como ventajas la identificación del riesgo por sexo y grupo etario, así como por regiones y departamentos en un periodo prolongado de tiempo, situación que describe un problema de salud pública poco explorado. Sin embargo, se reconoce como limitantes, el trabajar con fuentes de información secundaria, aspecto que genera problemas de calidad, registro y clasificación de los eventos, en ocasiones, de forma no apropiada ${ }^{44,45}$; adicionalmente, es posible que existan sub-reportes para este grupo de eventos en algunas regiones, dada la estigmatización de la conducta al interior de las familias como primer notificador del evento ${ }^{46}$. Tampoco esta información refleja en tiempo real la ocurrencia de dichos eventos; para el caso del suicidio, no es posible identificar factores potencialmente relacionados con la ideación e intento de suicidio o con registros que permitan el respectivo seguimiento.

\section{Conclusiones y recomendaciones}

En Colombia, aunque el riesgo de mortalidad por suicidio ha descendido, ha sido cuatro veces más alto en hombres que en mujeres, en hombres, adultos jóvenes y adultos mayores; las regiones del país con mayor riesgo de muerte han sido aquellas con departamentos en zonas centrales y orientales. Con base a estos resultados, en el futuro mediato deben establecerse investigaciones que permitan explorar a profundidad los determinantes y/o factores de riesgo potencialmente asociados a estos comportamientos, tanto por sexo/género, por grupos de edad, como en las regiones con mayor riesgo de muerte por este evento prevenible.

A pesar que el comportamiento nacional y regional de las tasas de suicidio en Colombia son menores a las incidencias internacionales ${ }^{25,26}$, en el país, con base a la legislación reciente ${ }^{47}$, se hace necesario desde los niveles de atención primaria diseñar, implementar y monitorear estrategias, 
fundamentadas en el enfoque de derechos y atención integral en salud, orientadas a la detección de las personas con estados depresivos, con ideación suicida y/o con intentos de suicidio para brindar las herramientas que controlen y minimicen el riesgo de un evento auto-inflingido a personas con estas condiciones; estas intervenciones, idealmente, deben estar basadas en diseños cuasi-experimentales para identificar el posible impacto poblacional de las mismas.

\section{Referencias}

1. World Health Organization (WHO). Informe mudial sobre la violencia y la salud 2002. [citado 2016 Mayo 30]. Disponible en: http://www.who.int/violence_injury_prevention/violence/world_report/es/summary_ es.pdf

2. Organización Panamericana de la Salud (OPAS). Vigilancia de la Salud y Prevención y Control de Enfermedades Información y Análisis de Salud. Indicadores básicos de salud de las Américas para 2011-2012. Washington: OPAS; 2014.

3. Van Heeringen K, Bijttebier S, Desmyter S, Vervaet $\mathrm{M}$, Baeken C. Is there a neuroanatomical basis of the vulnerability to suicidal behavior? A coordinate-based meta-analysis of structural and functional MRI studies. Frontiers in Human Neuroscience 2014; 8:824.

4. Pérez-Olmos I, Ibáñez-Pinilla M, Reyes-Figueroa J, Atuesta-Fajardo J, Suárez-Díaz M. Factores Asociados al intento suicida e ideación suicida persistente en un Centro de Atención Primaria. Bogotá, 2004-2006. Revista de Salud Pública 2008; 10(3):374-85.

5. Doak MW, Nixon AC, Lupton DJ, Waring WS. Self-poisoning in older adults: patterns of drug ingestion and clinical outcomes. Age Ageing 2009; 38(4):407-411.

6. Minayo MCS, Cavalcante FG, Souza ER. Methodological proposal for studying suicide as a complex phenomenon. Cad Saude Publica [Internet]. 2006 Ago [citado 2016 Mayo 31]; 22(8):1587-1596. Disponible en: http://www.scielo.br/scielo.php?script=sci_arttext\&pid=S0102-311X2006000800007\&lng=es

7. Capron DW, Fitch K, Medley A, Blagg C, Mallott M, Joiner T. Role of anxiety sensitivity subfactors in suicidal ideation and suicide attempt history. Depress Anxiety 2012; 29(3):195-201.

8. Rihmer Z. Strategies of suicide prevention: focus on health care. J Affect Disord 1996; 39(2):83-91.

9. World Health Organization (WHO). Health statistics and information systems. Global Health Estimates (GHE). [citado 2016 Mayo 31]. Disponible en: http:// www.who.int/healthinfo/global_burden_disease/en/\#

\section{Colaboradores}

JM Rodríguez-Hernández: concepción, diseño, análisis de información y escritura del artículo. Geovane Mendienta-Izquierdo: diseño y escritura del artículo, búsqueda bibliográfica. A Rocha-Buelvas: análisis de la información, escritura de resultados y discusión. Arsenio Hidalgo-Troya: análisis de la información, escritura e interpretación de resultado.

10. Gómez-Restrepo C, Rodríguez N, Bohórquez A, Diazgranados N, Ospina-García MB, Fernández C. Factores asociados al intento de suicidio en la población colombiana Revista Colombiana de Psiqiatria 2002; (4):271-286

11. Peñaloza-Quintero RE, Salamanca-Balen N, Rodríguez-Garcia J, Beltrán-Villegas AR, Rodríguez-Hernandez JM. Estimación de carga de enfermedad para Colombia, 2010. Bogotá: Editorial Pontificia Universidad Javeriana; 2014.

12. Miettinen OS. Theoretical epidemiology: principles of ocurrence research in medicine. Nueva York: Wiley; 1985.

13. Organización Mundial de la Salud (OMS). Manual de codificación. CIE-10ES Diagnósticos. Geneva: Unidad técnica de codificación CIE-10-ES; 2008.

14. Colombia. Ministerio de Salud y Protección Social. Análisis de Situación de Salud según regiones Colombia. Bogotá: Ministerio de Salud y Protección Social; 2013.

15. Departamento Nacional de Estadíctica (DANE). Proyecciones nacionales y departamentales de población [Internet]. Proyecciones nacionales y departamentales de población. Bogotá: DANE; 2012. [citado 2016 Mayo 30]. Disponible en: http://dane.gov.co/index.php/estadisticas-regionales/informe-de-coyuntura-economica -regional-icer

16. Ahmad OB, Boschi-pinto C, Lopez AD. Age standardization of rates: A new WHO standard GPE. Discussion Paper Series: No . 31 EIP / GPE / EBD World Health Organization 2001. [citado 2016 Jun 4]. Disponible en: http://www.who.int/healthinfo/paper31.pdf

17. Piñeres-Petersen M, Pardo-Ramos C, Gamboa-Garay O, Hernández-Suárez G. Atlas de mortalidad de cáncer en Colombia. 3 ed. Bogotá: Instituto Nacional de Cancerología, Instituto Geográfico Agustín Codazzi (IGAC), Imprenta Nacional de Colombia; 2010.

18. Oleckno W. Epidemiology: concepts and methods. Illinois: Waveland Press; 2008. 
19. Dobson AJ, Barnett A. An introduction to generalized linear models. $3^{\text {th }}$ Edit. Chatfield C, editor. New York, Zidek: University of Bath, University of British Columbia; 2008. p. 1-219.

20. Vives-Brosa J. El diagnóstico de la sobredispersión en modelos de análisis de datos de recuento. Barcelona: Universitat Autònoma de Barcelona; 2002.

21. Peterson JJ, Cameron C, Trivedi PK. Regression Analysis of Count Data. Technometrics [Internet]. 1999. [citado 2016 Jun 05]; 41(4):371. Disponible en: http:// www.jstor.org/stable/1271358? origin $=$ crossref

22. Preti A, Miotto P. Seasonality in suicide: the influence of suicide method, gender and age of suicide distribution in Italy. Psyquiatric Res 1998; 81:219-223.

23. Maris RW. Suicide. Lancet 2002; 360(9329):319-326.

24. Gaxiola-Robles R, Bitzer-Quintero OK, García Gonzáles A, Celis-De la Rosa AJ. El estado civil y el suicidio en Baja California. Rev Med Inst Seguro Soc 2009; (47):383-386.

25. Krug E, Dahlberg L, Mercy J, Zwi A, Lozano R. La violencia, un problema mundial de salud pública. In: Informe mundial sobre la violencia y la salud. Washington: Organización Panamericana de la Salud; 2003. Págs. 1-21. [citado 2016 Jun 05]. Disponible en: http://apps. who.int/iris/bitstream/10665/112670/1/9275315884_ spa.pdf

26. Mendieta-Izquierdo G. Cuerpo masculino: un análisis para la salud pública. Rev. Fac. Nac. Salud Pública [Internet]. 2015 Abr [citado 2016 Jun 05]; 33(1):100-110. Disponible en: http://www.scielo.org.co/scielo.php?scrip$\mathrm{t}=$ sci_arttext\&pid=S0120=386-2015000100013X\&lnges.

27. Kripper CE, Sapag JC. Capital social y salud en América Latina y el Caribe: una revisión sistemática. Rev Panam Salud Pública 2009; 25(2):162-170.

28. Fässberg MM, Cheung G, Canetto SS, Erlangsen A, Lapierre S, Lindner R, Draper B, Gallo JJ, Wong C, Wu J, Duberstein P, Wærn M. A systematic review of physical illness, functional disability, and suicidal behaviour among older adults. Aging Ment Health 2016; 20(2):166-194.

29. Callejo J. Cien años después. Los rasgos de modernidad del suicidio. Reis 1998; 81:73-98.

30. Collins R. Cuatro tradiciones sociológicas. México: UAM; 1996.

31. Desapriya E, Iwase N. Stigma of mental illness in Japan. Lancet 2002; 359(8320):1866-1866.

32. Wasserman D, Värnik A, Dankowicz M, Eklund G. Suicide prevention effects of perestroika in the former USSR: the role of alcohol restriction. Acta Psychiatr Scand 1998; 394(Supl.):1-44.

33. Campo-Arias A, Herazo E. Asociación entre desigualdad y tasa de suicidio (1994-2013). Revista Colombiana de Psiquiatría 2015; 44:28-32.

34. Campo-Arias A, Herazo E. Pobreza, desigualdad y tasa de suicidio en Colombia, 2012. Duazary 2014; 11(2):126-130.

35. Morgenstern H. Ecologic studies in epidemiology: concepts, Principles and methods. Annual Review of Public Health 1995; 16:61-81.

36. Londoño DA, Ortega Lenis D, Olaya Ochoa J. Modelación del número de homicidios vía regresión de Poisson. Heurística 2009; 16:81-90.
37. Chaparro-Narváez P, Castañeda-Orjuela C, León-Quevedo DD, Jiménez W. Análisis descriptivo del suicidio en Colombia, 1998-2012. In: Instituto Nacional de Salud, editor. Carga de enfermedad por enfermedades crónicas no transimisbles y discapacidad en Colombia. Quinta. Bogotá, Colombia: Ministerio de Salud y Protección Social; 2015. p. 129-148.

38. Colombia. Ministerio de Salud y Protección Social, Colciencias, Pontificia Universidad Javeriana. Encuesta Nacional de Salud Mental 2015. Tomo 1. p. 343. [Internet]. 2015. [citado 2016 Jun 5]. Disponible en http:// www.odc.gov.co/portals/1/publicaciones/pdf/consumo/estudios/nacionales/co031102015-salud_mental_tomoi.pdf

39. Campo-Arias A, Herazo E. El complejo estigma-discriminación asociado a trastorno mental como factor de riesgo de suicidio. Revista Colombiana de Psiquiatría 2015; 44:243-250.

40. Yang B, Lester D. Recalculating the Economic Cost of Suicide. Death Studies 2007; 31(4):351-361

41. Schulberg HC, Bruce ML, Lee PW, Williams Junior JW, Dietrich AJ. Preventing suicide in primary care patients: the primary care physician's role. Gen Hosp Psychiatry 2004; 26(5):337-345.

42. Shah A. Does improvement in the treatment of those who attempt suicide contribute to a reduction in elderly suicide rates in England? International Psychogeriatrics 2009; 21(4):768-773.

43. Organización Mundial dela Salud(OMS), Organización Panamericana de la Salud (OPAS). Prevención del Suicidio. Un imperativo Global. Washington: OMS; 2014. [citado 2016 Jun 5]. Disponible en: http://apps.who. int/iris/bitstream/10665/136083/1/9789275318508_ spa.pdf

44. Comstock GW. Further comments on problems in death certification. Am J Epidemiol 1986; 124(2):180181.

45. Celis A. El peatón lesionado en accidentes de tráfico de vehículo de motor: Mortalidad en México: 1985-1996. Gac Med Mex 1999; 135(3):353-358.

46. Rodríguez-Téllez N. Patología Forense. Un enfoque centrado en derechos humanos. In: Alzate JP, Eslava-Smalbach J, Urrego-Mendoza ZC, Urrego-Mendoza DZ, Rocha-Buelvas A, organizadores. Epidemiologia de la Violencia. Bogotá: Universidad Nacional de Colombia-Instituto de Medicina Legal y Ciencias Forenses; 2014. Tomo I; Parte II. Pág. 135-167.

47. Congreso de Colombia. Ley Estatutaria de Salud 1751 de febrero de 2015. Por medio de la cual se regula el derecho fundamental a la salud y se dictan otras disposiciones. [citado 2016 Jun 5]. Disponible en: https:// www.minsalud.gov.co/Normatividad_Nuevo/Ley\%20 1751\%20de\%202015.pdf

Artigo apresentado 23/03/2016

Aprovado em 25/10/2016

Versão final apresentada em 27/10/2016 